Original paper

\title{
Evaluation of early radiation DNA damage in a fractal cell nucleus model using Geant4-DNA
}

\author{
Dousatsu Sakata $^{\mathrm{a}, 1}$, Nathanael Lampe ${ }^{\mathrm{c}, \mathrm{d}, 1}$, Mathieu Karamitros ${ }^{\mathrm{e}, 1}$, Ioanna Kyriakou ${ }^{\mathrm{f}}$, Oleg Belov ${ }^{\mathrm{g}}$, \\ Mario A. Bernal $^{\mathrm{h}}$, David Bolst ${ }^{\mathrm{a}}$, Marie-Claude Bordage ${ }^{\mathrm{i}, \mathrm{j}}$, Vincent Breton ${ }^{\mathrm{b}}$, Jeremy M.C. Brown ${ }^{\mathrm{k}}$, \\ Ziad Francis, $^{1}$ Vladimir Ivanchenko ${ }^{\mathrm{m}, \mathrm{n}}$, Sylvain Meylan ${ }^{\mathrm{o}, \mathrm{p}}$, Koichi Murakami ${ }^{\mathrm{q}}$, Shogo Okada ${ }^{\mathrm{q}}$, \\ Ivan Petrovic $^{\mathrm{r}}$, Aleksandra Ristic-Fira ${ }^{\mathrm{r}}$, Giovanni Santin ${ }^{\mathrm{s}}$, David Sarramia ${ }^{\mathrm{b}}$, Takashi Sasaki ${ }^{\mathrm{q}}$, \\ Wook-Geun Shin $^{\mathrm{c}, \mathrm{d}}$, Nicolas Tang ${ }^{\mathrm{o}}$, Hoang N. Tran ${ }^{\mathrm{t}, \mathrm{u}}$, Carmen Villagrasa ${ }^{\mathrm{o}}$, Dimitris Emfietzoglou ${ }^{\mathrm{f}}$, \\ Petteri Nieminen ${ }^{\mathrm{s}}$, Susanna Guatelli ${ }^{\mathrm{a}}$, Sebastien Incerti ${ }^{\mathrm{c}, \mathrm{d}, *}$
}

${ }^{a}$ Centre For Medical Radiation Physics, University of Wollongong, Wollongong, Australia

${ }^{\mathrm{b}}$ Université Clermont Auvergne, CNRS/IN2P3, LPC, F-63000 Clermont-Ferrand, France

${ }^{\text {c } C N R S, ~ I N 2 P 3, ~ C E N B G, ~ U M R ~ 5797, ~ G r a d i g n a n, ~ F r a n c e ~}$

${ }^{\mathrm{d}}$ Université de Bordeaux, CENBG, UMR 5797, Gradignan, France

${ }^{\mathrm{e}}$ Bordeaux, France

${ }^{\mathrm{f}}$ Medical Physics Laboratory, University of Ioannina, Medical School, GR-45110 Ioannina, Greece

${ }^{\mathrm{g}}$ Joint Institute for Nuclear Research, Dubna, Russia

${ }^{\mathrm{h}}$ Instituto de Fisica Gleb Wataghin, Universidade Estadual de Campinas, Campinas, SP, Brazil

i INSERM, Université Paul Sabatier, UMR 1037, CRCT, Toulouse, France

${ }^{\mathrm{j}}$ Université Toulouse III-Paul Sabatier, UMR 1037, CRCT, Toulouse, France

${ }^{\mathrm{k}}$ Radiation Science and Technology, Delft University of Technology, Delft, The Netherlands

${ }^{1}$ Department of Physics, Faculty of Sciences, Université Saint Joseph, Beirut, Lebanon

${ }^{\mathrm{m}}$ Geant4 Associates International Ltd, Hebden Bridge, UK

${ }^{\mathrm{n}}$ Tomsk State University, Tomsk, Russia

${ }^{\circ}$ IRSN, Institut de Radioprotection et de Sûreté Nucléaire, 92262 Fontenay-aux-Roses, France

${ }^{\mathrm{P}}$ SymAlgo Technologies, Paris, France

${ }^{\mathrm{q}} \mathrm{KEK}$, Tsukuba, Japan

${ }^{\mathrm{r}}$ Vinca Institute of Nuclear Science, University of Belgrade, Belgrade, Serbia

${ }^{s}$ ESA-ESTEC, Noordwijk, The Netherlands

${ }^{\mathrm{t}}$ Division of Nuclear Physics, Advanced Institute of Materials Science, Ton Duc Thang University, Ho Chi Minh City, Viet Nam

${ }^{u}$ Faculty of Applied Sciences, Ton Duc Thang University, Ho Chi Minh City, Viet Nam

\section{A R T I C L E I N F O}

\section{Keywords:}

Geant4-DNA

Monte Carlo simulation

DNA damage

\begin{abstract}
A B S T R A C T
The advancement of multidisciplinary research fields dealing with ionising radiation induced biological damage - radiobiology, radiation physics, radiation protection and, in particular, medical physics - requires a clear mechanistic understanding of how cellular damage is induced by ionising radiation. Monte Carlo (MC) simulations provide a promising approach for the mechanistic simulation of radiation transport and radiation chemistry, towards the in silico simulation of early biological damage. We have recently developed a fully integrated MC simulation that calculates early single strand breaks (SSBs) and double strand breaks (DSBs) in a fractal chromatin based human cell nucleus model. The results of this simulation are almost equivalent to past MC simulations when considering direct/indirect strand break fraction, DSB yields and fragment distribution. The simulation results agree with experimental data on DSB yields within $13.6 \%$ on average and fragment distributions agree within an average of $34.8 \%$.
\end{abstract}

\footnotetext{
* Corresponding author at: CNRS, IN2P3, CENBG, UMR 5797, Gradignan, France.

E-mail address: incerti@cenbg.in2p3.fr (S. Incerti).

${ }^{1}$ These authors contributed equally to this work.
} 
(a)

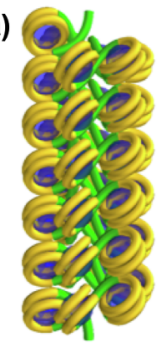

(b)

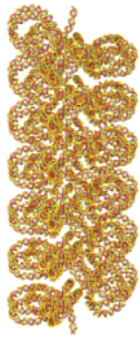

(c)
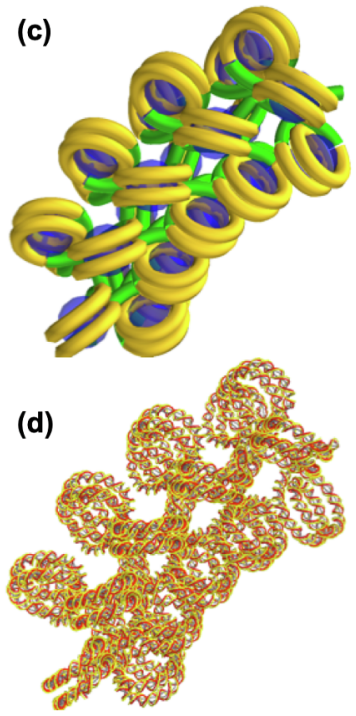

(e)

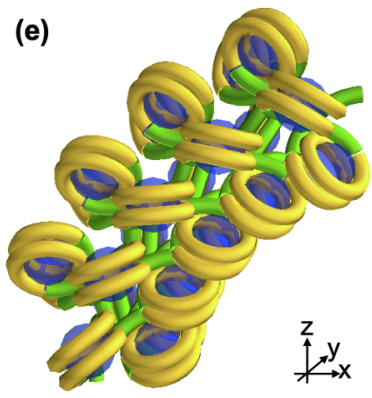

(f)

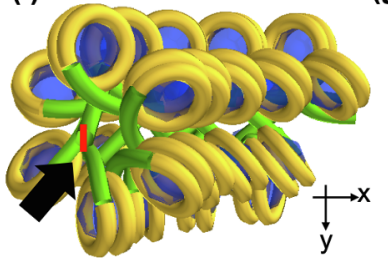

(g)

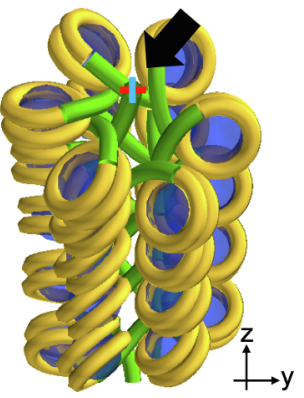

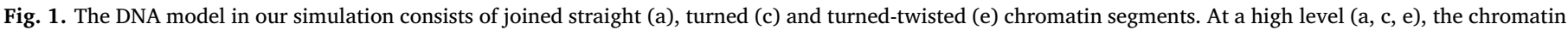

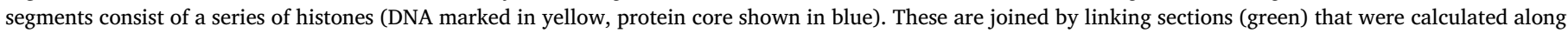

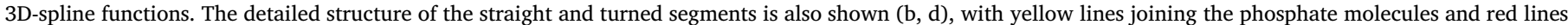

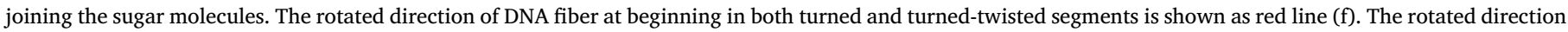

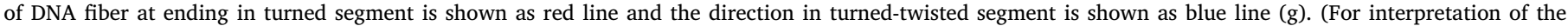
references to colour in this figure legend, the reader is referred to the web version of this article.)

\section{Introduction}

The advancement of multidisciplinary research fields which study the biological damage from ionising radiation - such as: radiobiology, radiation physics, radiation protection and, in particular, medical physics - requires a clear mechanistic understanding of how cellular damage is induced by ionising radiation [1-4]. Understanding and modeling the mechanisms of such radiation induced DNA damage is still challenging due to their complexity. Biomolecules which compose DNA can be damaged not only directly by radiation itself but also by radiolysis byproducts such as hydroxyl radicals. An increased understanding of the damage mechanisms from radiation will allow the radiation risk to be more accurately predicted in particular for radiation therapy, for radiation protection, and during space missions.

Monte Carlo (MC) simulations allow mechanistic models of radiation transport and radiation chemistry to be validated against experimental measurements of radiation damage, by simulating the entire process of early radiation damage induction in silico $[5,6]$. Over the last couple of decades, many research groups have developed MC track structure codes for radiobiology-related simulations such as KURBUC [7-9], PARTRAC [10-13] and Geant4-DNA based MC code TOPAS-nBio [14] (another comprehensive review can be found in reference [15]).

Recently, a fibroblast cell nucleus irradiated by protons was simulated using Geant4-DNA based on a step-by-step simulation of water radiolysis [16]. A similar approach was followed in another work that used an atomistic DNA model up to the chromatin fiber level [17]. The Geant4-DNA track structure code [18-21], a low-energy extension of the Geant4 general purpose MC simulation toolkit was used for this work [22-24]. These MC simulation codes are able to simulate DNA damage caused by physical processes (direct damage) such as ionisation and excitation, as well as the chemically-induced (indirect) DNA damage that arises when radiation-induced radicals created in water react with the DNA. Using chemical models based on the Independent Reaction Time (IRT) method [25], early DNA damage simulations using Geant4-DNA were further benchmarked in a simple geometry [26], before being extended to Escherichia coli (E. coli) bacterial cell $[27,28]$. In this paper, we have extended this application to simulate more complex DNA geometries as found in human cells. Using a continuous chromatin geometry, the early DNA damage arising from proton irradiation of a cell nucleus was simulated to determine the number of single strand breaks (SSBs), double strand breaks (DSBs) and DNA fragment distributions. These simulated results are compared to experimental data and previous literature simulation results.

\section{Materials and methods}

We developed a fully integrated application to simulate early DNA damage mechanistically by extending the Geant4-DNA application for a human cell nucleus as first presented by Lampe et al. [26-28]. The integrated simulation calculates early SSBs and DSBs in an irradiated cell nucleus from direct and indirect damage in a single application. This application consists of a beam incident upon the detailed geometry of the cell nucleus, with both the chemistry and physics being described. These functionalities can be fully controlled through a macro file. All physical and chemical processes and damage definitions remain the same as defined in the previous work done by Lampe et al. [26-28], with the exception of the addition of histones, which were defined as spheres, each with a $25 \AA$ radius within which all chemical radicals were considered to be immediately scavenged. In this section we briefly summarise the modeling of the geometry, physics and chemistry, as well as early damage calculations.

\subsection{Nucleus geometry}

The DNA fiber can be considered as being composed of phosphate $\left(\mathrm{H}_{3} \mathrm{PO}_{4}\right)$ and deoxyribose $\left(\mathrm{C}_{5} \mathrm{H}_{10} \mathrm{O}_{4}\right)$ molecules forming a backbone that supports the nucleotide bases, guanine $\left(\mathrm{C}_{5} \mathrm{H}_{5} \mathrm{~N}_{5} \mathrm{O}\right)$, adenine $\left(\mathrm{C}_{5} \mathrm{H}_{5} \mathrm{~N}_{5}\right)$, cytosine $\left(\mathrm{C}_{4} \mathrm{H}_{5} \mathrm{~N}_{3} \mathrm{O}\right)$ and thymine $\left(\mathrm{C}_{5} \mathrm{H}_{6} \mathrm{~N}_{2} \mathrm{O}_{2}\right)[27,28]$. We approximated the phosphate and sugar molecules as spheres, whilst the bases, due to their flatter shape, were interpreted as ellipsoids $[27,28]$. In the cell nucleus, the DNA fiber is folded by histones compactly forming chromatin fiber. Our Geant4-DNA application builds a chromatin fiber out of smaller $75 \mathrm{~nm}$ cubes repeating straight (shown in Fig. 1(a, b)) and $90^{\circ}$ turned (shown in Fig. 1(c, d)) chromatin segments that join together to form a $14.2 \mu \mathrm{m}$ long ellipsoidal continuous segment (shown in the left panel of Fig. 3). 
Beginning from the same geometry for a single base pair as used in our previous works $[27,28]$, we built turned and straight chromatin segments to create the geometry. The turned segments contained 26 histones and 5024 base pairs, and the straight segments contained 38 histones and 7331 base pairs (Fig. 1). Histones were linked by DNA sections interpolated between the end of one histone and the beginning of the next one using spline functions.

In order to ensure that the DNA helix joins smoothly when rotating, we also created variants of these straight and turned DNA structures, having the same number of base pairs and histones, where the chain went through an additional $90^{\circ}$ rotation, which we called turnedtwisted segments in Fig. 1(e). The difference between turned and turned-twisted is illustrated in Fig. 1(g). In both the twisted and nontwisted cases, the DNA strand begins aligned with the y-axis (red line in Fig. 1(f)). In the normal turned strand, the DNA is aligned when leaving the chromatin fiber along the y-axis (red line in Fig. $1(\mathrm{~g})$ ), while in the twisted case, it is aligned with the z-axis (blue line in Fig. 1(g)). Using both these DNA segments (turned with and without twisting) allows the DNA to be joined continuously between segments.

The continuous chain was defined by taking a basic Hilbert curve and iterating it as a fractal 8 times, creating a fractal that could be broken into $1.68 \times 10^{7}$ cubic regions of straight and turned chromatin sections. An ellipsoid with dimensions $(14.2 \mu \mathrm{m} \times 14.2 \mu \mathrm{m} \times 5 \mu \mathrm{m})$ was used to define the cell nucleus. Cubic regions from the Hilbert curve falling outside this region were excluded from the simulation, leaving $6.4 \mathrm{Gbp}$ in total (bp stands for base pair). The overall bp density is $\sim 0.012 \mathrm{bp} / \mathrm{nm}^{3}$ (typical bp density of a human cell nucleus is $\sim 0.015 \mathrm{bp} / \mathrm{nm}^{3}$ [29], compared to $\sim 0.008 \mathrm{bp} / \mathrm{nm}^{3}$ in the previous Geant4-DNA simulations [16]). This process of using a fractal approach to seed a mostly continuous nucleus is shown in Fig. 2, and the whole cell used for this work is shown in the left panel of Fig. 3.

The DNA damage to the cell was studied for various incident proton energies, with the cell nucleus being irradiated with monoenergetic beams between 0.1 and $50 \mathrm{MeV}$, fired perpendicular to the $14.2 \mu \mathrm{m}$ circular plane of the cell-nucleus's equator, as shown in the right panel of Fig. 3.

\subsection{Particle transportation and calculation of direct damage}

Geant4-DNA provides track structure models for protons, our primary particle, neutral hydrogen atoms and all secondary particles electrons, photons. For protons, elastic scattering, excitation and ionisation processes are modelled, as well as charge-exchange, which becomes increasingly important at low energies [30]. Geant4-DNA provides three alternative sets of physics models for electron transportation in liquid water: (1) a set based on the partial-wave formalism for elastic scattering that also includes ionisation and excitation inelastic cross sections calculated from the dielectric model of Emfietzoglou [31,32], (2) a set based on the screened Rutherford elastic scattering formulas with inelastic scattering processes based on an improved dielectric function [33,34] and (3) the well-known CPA100 MC set for both elastic and inelastic scattering processes [35]. In this work, we use the so-called Geant4-DNA physics option 4 (corresponding to (2)), which offers a better performance for electron transport in the proton energy range under investigation [33-36]. For photon transport, the Livermore low energy photon library available in Geant4 was utilised.

In order to calculate direct damage the same methodology as used in the PARTRAC simulation code was adopted [11]. For defining DNA strand breaks, all energy deposited within $4.5 \AA$ of the centre of a DNA sugar or phosphate molecule was allocated to the closest strand molecule. After each simulation run, the total cumulative energy deposited in this region was used to determine the probability that a strand break occurred along the chain, based on a linearly increasing probability distribution, where there was a $0 \%$ probability a break occurred when less than $5 \mathrm{eV}$ was deposited, and a $100 \%$ probability a break occurred when over $37.5 \mathrm{eV}$ was deposited in the sugar phosphate moiety.

\subsection{Water radiolysis and indirect damage}

During irradiation, molecular species are created when particles interact in water [37-39]. This work uses an Independent Reaction Time (IRT) method to simulate chemistry $[28,40]$ rather than the stepby-step (SBS) method available in the public version of Geant4-DNA $[41,42]$, as introduced in past works $[26,27]$. Indirect damage depends heavily on the value chosen for the likelihood of a chemical reaction

\section{A. Seed Hilbert Curve}
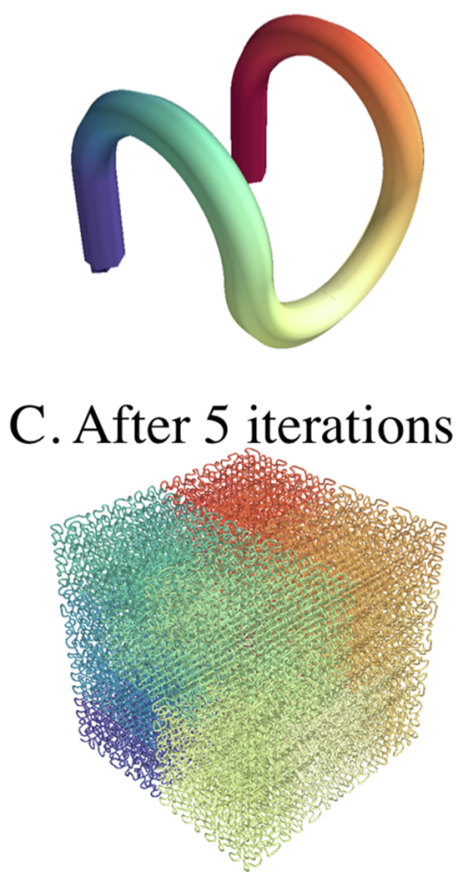

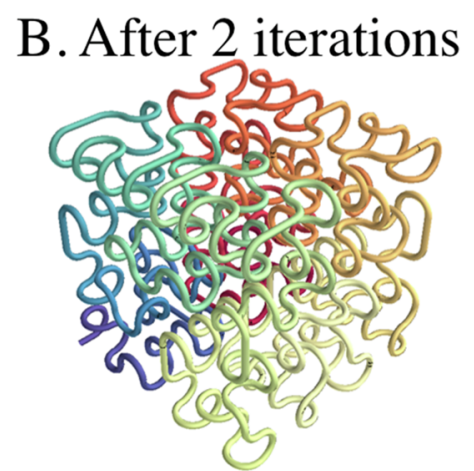

D. Curve + Spherical Mask

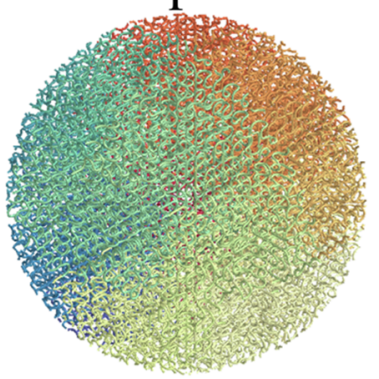

Fig. 2. Fractals can be used to seed a mostly continuous DNA structure for the cell nucleus. The colour varies continuously from blue to white to red from one end of the chain to the other. A fractal Hilbert Curve (A) is used to seed the continuous curve of the DNA, which is iterated (B). When the DNA is sufficiently dense (C), a mask is applied to create a nucleus of the desired size (D). Each curved and straight section making up the curve is then replaced with chromatin in the simulation (not shown). For this work, we iterate the base Hilbert Curve eight times before applying an elliptical mask (see the left panel of Fig. 3). (For interpretation of the references to colour in this figure legend, the reader is referred to the web version of this article.) 

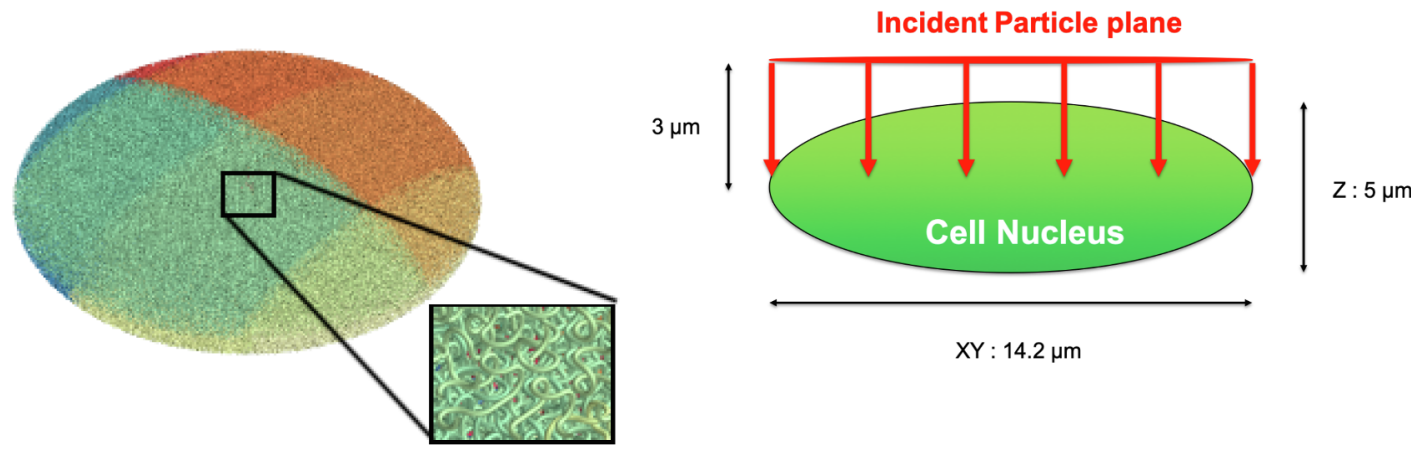

$X Y: 14.2 \mu \mathrm{m}$

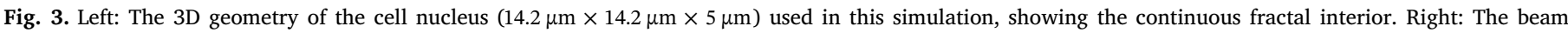

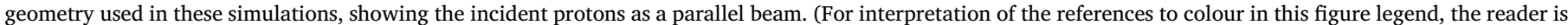
referred to the web version of this article.)

between a hydroxyl radical and the sugar phosphate backbone leading to a single strand break (SSB). Following past studies [26], we assign a probability of $40 \%$ to induce a strand break. Additionally, we kill all chemical species created more than $4.5 \mathrm{~nm}$ away from any DNA molecules, as they will likely be scavenged. This range cut is equivalent to the maximum diffusion distance of hydroxyl radical at $2.5 \mathrm{~ns}$, hence all simulations are stopped at $2.5 \mathrm{~ns}$ after diffusion started. Chemically, we model histones as perfect scavengers. This means that any free radical species that enters a histone region (blue regions in Fig. 1) will be killed [11].

\subsection{Damage scoring and experimental results}

In our present work, all strand breaks (SBs) are classified either direct SB or indirect SB. The number of DSBs is of particular interest when quantifying damage to the DNA. We follow the classification scheme of DSBs proposed by Nikjoo et al. [8] to determine DSBs which are considered to be at least two strand breaks on opposite strands within $10 \mathrm{bp}$ of each other. The evaluated DNA damage is compared to experimental results [43-46] and previous MC simulations (KURBUC, PARTRAC, Geant4-DNA-2017) [8,11,16].

Experimentally, there are mainly two ways to evaluate DSB yields. The first is based on detection of foci representing the accumulation of proteins to repair DSBs [47]. The second is based on the separation of DNA fragments through agarose gel electrophoresis (AGE) [48] and/or pulsed-field gel electrophoresis (PFGE) [49] (usually scaled by the energy deposited in Gy and the length of DNA). The foci measurement is becoming a standard to evaluate radiation induced DNA damage, however, the relation between foci yield and number of DSBs is still unclear. Hence, in this paper, experimental data by AGE and PFGE have been compared to the simulated number of DSBs as benchmark. As AGE and PFGE cannot detect small DNA fragments (shorter than 5-25 kbp) because of their loss during the standard lysis procedure of cells embedded in gel plugs [44], we calculate not only the total number of DSBs but also that of "distant" DSBs, those that are separated by at least $5 \mathrm{kbp}$ or $10 \mathrm{kbp}$ gaps between two DSBs. The DSB yields are presented as a function of unrestricted linear energy transfer $\left(\mathrm{LET}_{\infty}\right)$, which is the electronic stopping power as recommended by the ICRU [50]. Additionally, we have calculated the fragment (gap between two DSBs) size distribution and compared it to experimental results [51]. We evaluate the accuracy of the simulations by calculating the average percentage error of deviation from the experimental data set as $\sum_{i=1}^{n}\left|\left(V_{i}^{\text {sim }}-V_{i}^{\text {exp }}\right) / V_{i}^{\text {exp }}\right| / n_{\text {data }} \times 100$, where $V_{i}^{\text {sim }}$ is the $i$ th simulated value, $V_{i}^{\exp }$ is the ith experimental value and $n_{\text {data }}$ is the number of experimental data.

\section{Results and discussion}

The main goals of the application developed in this work are to create a fractal based DNA geometry having a more realistic base pair density $\sim 0.012 \mathrm{bp} / \mathrm{nm}^{3}$ (the typical bp density of a human cell nucleus is $\sim 0.015 \mathrm{bp} / \mathrm{nm}^{3}$ [29], compared to $\sim 0.008 \mathrm{bp} / \mathrm{nm}^{3}$ in the previous Geant4-DNA simulations [16]), and to simulate diffusion of radiolysis products based on IRT method. In addition, we introduced the linearly increasing probability model for direct damage induction which was proposed initially in PARTRAC simulations.

The total numbers of strand breaks (SBs) are plotted in Fig. 4 as a function of LET, with all strand breaks categorized into either direct or indirect damage. In this work, the number of strand breaks and their fraction of direct to indirect, obtained in this work, are similar to the results obtained by the PARTRAC code [11].

The yield of direct damage is almost flat across the LET range, indicating that the number of direct strand breaks is not sensitive to LET for the present proton irradiation conditions. As it is well-known the number of direct DSB increases with LET due to proximity of ionizations whereas the number of direct SSB decreases with LET (since more SSB participate in forming DSB). The absolute number of direct breaks depends largely on the density of the number of bases and the energy deposited in the track of the incident protons [11]. In the previous Geant4-DNA simulations [16], a different direct damage model (step function probability from $17.5 \mathrm{eV}$ ) had been used, and the sensitive volume (nucleotide volume) for scoring energy deposition was smaller than the one used in this work. If this step function probability is applied in the present work, the number of direct damage increases

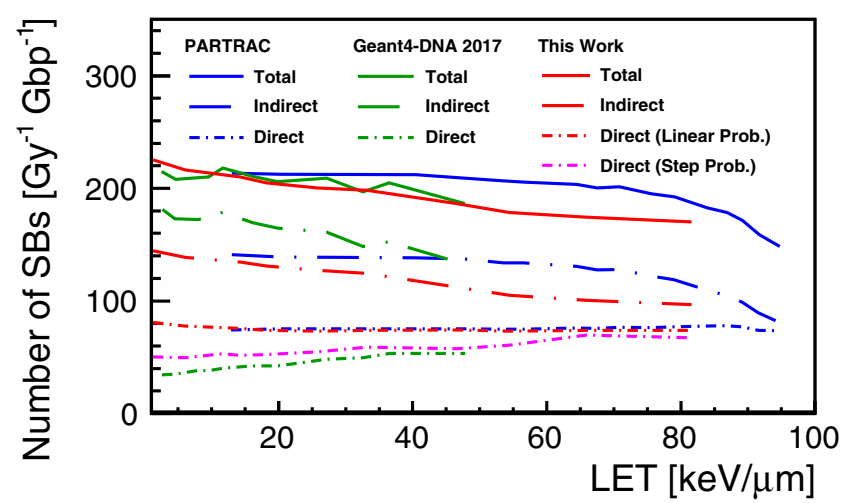

Fig. 4. Number of strand breaks (SBs) per Gy and per Gbp induced by protons in a cell nucleus as a function of LET. The solid lines show the total SB yield; the long dot-dashed lines show the indirect SB yield; the short dot dashed lines show the direct SB yield. For this work, two types of probability models have been applied, the linear probability model (default model in this work) is shown as red, and the step function probability model (at $17.5 \mathrm{eV}$ ) is shown as magenta for comparison with the previous Geant4-DNA simulations (Geant4-DNA 2017, in green) [16]. PARTRAC simulations are shown as well, in blue [11]. 
somewhat with LET (see magenta curve of Fig. 4). This is also observed in previous Geant4-DNA simulations (see short dot dashed green curve in Fig. 4, Geant4-DNA 2017) [16], although the values obtained in this work are larger than Geant4-DNA 2017 values.

Unlike direct damage, the indirect SB yield shows strong LET dependence with the indirect SB yield decreasing with increasing LET. At high LET, the incident protons deposit their energy locally, quickly slowing down. During this phase, protons produce many radiolytic products as well as secondary particles with LET in the range $10-20 \mathrm{keV} / \mu \mathrm{m}$. In the case where the density of radiolytic products is high, the chemical species interact with each other rather than interacting with DNA components, leading to neutralization. Compared to the previous Geant4-DNA simulations (Geant4-DNA 2017) [16], the LET trend is similar but smaller indirect SB yield was simulated, even if very similar indirect damage model has been used. Both IRT and SBS methods are usually equivalent if fine time steps are used, although the IRT method should be significantly faster to calculate diffusion of radiolytic products. In future works, we would like to explore the impact of different geometry implementations in the exact same chemistry and physics pipeline to isolate the cause of these differences.

Fig. 5 shows the simulated DSB and SSB/DSB ratio as a function of LET, and LET values corresponding to the primary proton energy, including previous simulation results $[9,11,16]$ and experimental results [43-46]. As explained in Section 2.4, to account for experimental bias on the DSB yield, we calculate the total DSB yield (dotted red line), $5 \mathrm{kbp}$ distant DSB yield (dashed red line) and 10kbp distant DSB yield (solid red line).

For both total DSB and distant DSB yields, the simulations show similar trends to PARTRAC [11] and agree with experimental data [43] on DSB yields with a $13.6 \%$ average error. At low LETs, the total DSB yield increases with the increase of LET up to $60 \mathrm{keV} / \mu \mathrm{m}$, then gradually decreases. At the same time, the distant DSBs start to decrease beyond $30 \mathrm{keV} / \mu \mathrm{m}$, largely due to the increase in production of small fragments. In terms of SSB/DSB ratio, the ratio decreases continuously
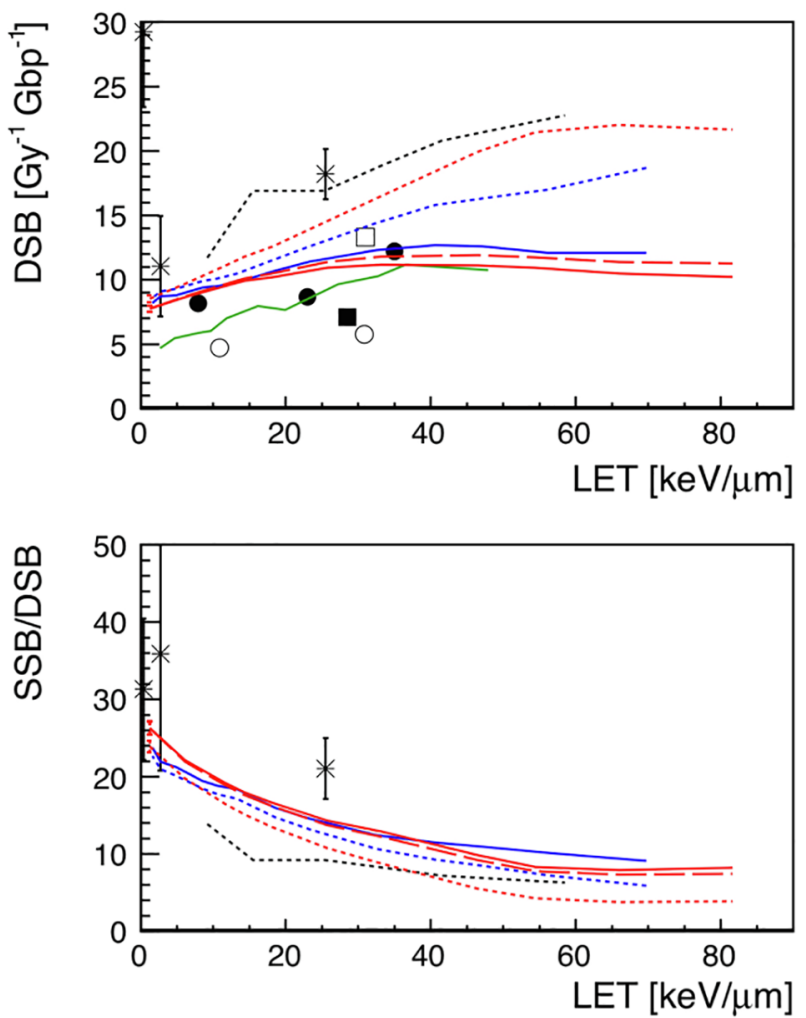

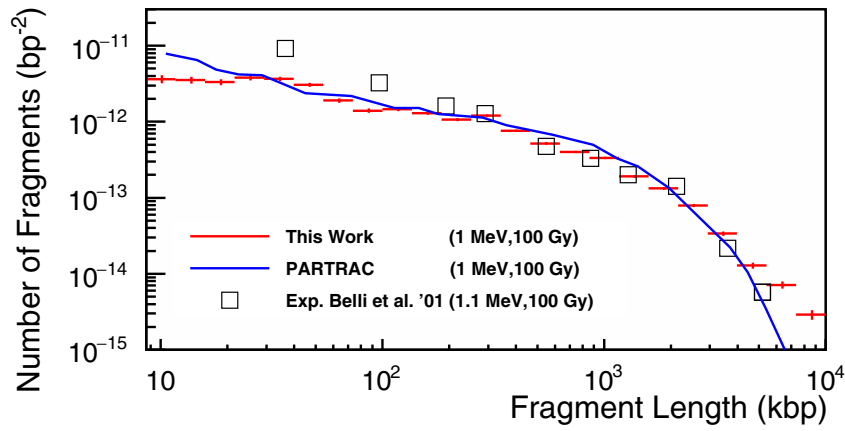

Fig. 6. Histogram of the fragment distribution following a simulated $100 \mathrm{~Gy}$ irradiation of the nucleus with $1 \mathrm{MeV}$ protons. The number of fragments is normalised by the total number of base pairs. The simulated fragment distribution is compared to PARTRAC simulations and to measurements from a hamster cell (V79) under a $100 \mathrm{~Gy}$ irradiation by a $3 \mathrm{MeV}$ incident proton beam $[11,51]$. After interacting with the cell container, the protons have an average exit energy of $1.1 \mathrm{MeV}$.

as a function of LET, and is in close agreement with PARTRAC. We note that the uncertainties of experimental results (e.g., cell line, experimental procedure, temperature, beam properties and so on) are still large, hence underlining the need for more experimental data and associated simulations.

The fragment distribution following a $100 \mathrm{~Gy}$ irradiation of a cell by $1 \mathrm{MeV}$ protons is shown in Fig. 6 . We find that the simulation produces a very similar fragment distribution from $20 \mathrm{kbp}$ to $5 \mathrm{Mbp}$ to PARTRAC simulation [11] and that the results agree with experimental data [51] with a $34.8 \%$ average error, and relatively better than PARTRAC from $200 \mathrm{kbp}$ to $5 \mathrm{Mbp}$. It ought to be noted that the DSB yield in this experimental measurement for a hamster cell (V79) is higher than in the other experiments shown in the top left panel of Fig. 5, hence the results may be slightly biased compared to other experiments in human
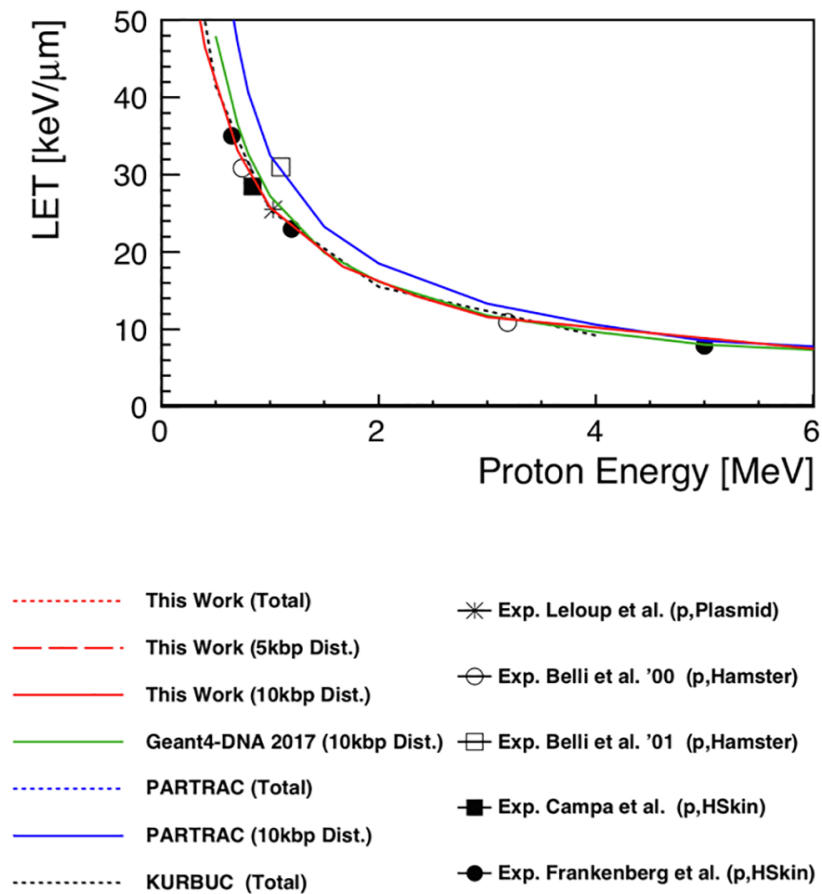

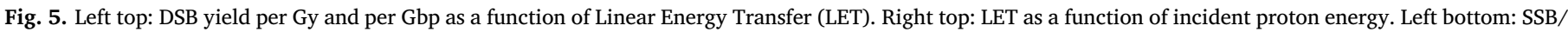
DSB ratio as a function of LET. Geant4-DNA 2017 [16], PARTRAC [11] and KURBUC [9] simulations and experimental data [43-46] are shown as well. 
fibroblast cells. The length of fragments decreases with the increase of the number of DSBs. Hence if the total number of DSBs is actually lower, then the number of long fragments is expected to be larger, as simulated with Geant4-DNA.

\section{Conclusion}

To help understanding the mechanisms of DNA damage induction from ionising radiation, we have developed a fully integrated Monte Carlo simulation code based on the Geant4-DNA toolkit that calculates SSBs and DSBs in a fractal chromatin based human cell nucleus model. In the presented work, the cell nucleus model proposes a more realistic base pair density $\left(\sim 0.012 \mathrm{bp} / \mathrm{nm}^{3}\right)$ and the IRT method has been used to compute the diffusion of the radiolytic products, allowing faster simulations. The results of this simulation are almost equivalent to past PARTRAC simulations when considering direct/indirect SB fraction, DSB yields and fragment distribution. The simulation results agree with experimental data on DSB yields within $13.6 \%$ on average and fragment distributions agree within an average of $34.8 \%$.

\section{Acknowledgements}

We would like to acknowledge the financial support from the CNRS PICS \#7340 and PICS \#8235 France - Greece, from the CNRS PICS \#8070 France - Serbia, the IdEx Bordeaux University - France International Post-doctorates program in the framework of the "FranceJapan Particle Physics Laboratory" International Associated Laboratory, and the IdEx Bordeaux University - France - International Doctorates program in the framework of the "France-Korea Particle Physics Laboratory" International Associated Laboratory (2017-2020) S. Guatelli, D. Sakata, S. Incerti, I. Kyriakou and D. Emfietzoglou acknowledge financial support from the Australian Research Council, ARC DP170100967. I. Kyriakou and D. Emfietzoglou acknowledge financial support from ESA (Contract No. 4000112863/14/NL/HB). M. A. Bernal thanks the CNPq for financing his research activities through the project 306775/2015-8, as well as the FAPESP foundation in Brazil, for the projects $2011 / 51594-2$ and $2015 / 21873-8$.

\section{References}

[1] Ying Z, et al. An expanded multi-scale Monte Carlo simulation method for personalized radiobiological effect estimation in radiotherapy: a feasibility study. Sci Rep 2017;7:45019.

[2] Suzuki K, et al. Low-dose radiation exposure and carcinogenesis. Jpn J Clin Oncol 2012;42(7):563-8

[3] Feinendegen LE, et al. Responses to Low doses of ionizing radiation in biological systems. Nonlin Biol Toxicol Med 2004;2(3):143-71.

[4] Moreno-Villanueva M, et al. Interplay of space radiation and microgravity in DNA damage and DNA damage response. NPJ Micrograv 2017;3:14.

[5] Nikjoo H, et al. Track structure in radiation biology: theory and applications. Int J Radiat Biol 1998;73(4):355-64.

[6] Nikjoo H, et al. Radiation track, DNA damage and response-a review. Rep Prog Phys 2016;79:116601.

[7] Uehara S, et al. Cross-section of water vapour for the Monte Carlo electrons track structure code from $10 \mathrm{eV}$ to the MeV region. Phys Med Biol 1993;38:1841-58.

[8] Nikjoo H, et al. Computational modeling of low-energy electron-induced DNA damage by early physical and chemical events. Int J Radiat Biol 1997;71(5):467-83.

[9] Nikjoo $\mathrm{H}$, et al. Computational approach for determining the spectrum of DNA damage induced by ionizing radiation. Radiat Res 2001;156:577-83.

[10] Friedland W, et al. Monte Carlo simulation of the production of short DNA fragments by low-linear energy transfer radiation using higher-order DNA models. Radiat Res 1998;150:170-82.

[11] Friedland W, et al. Simulation of DNA damage after proton irradiation. Radiat Res 2003;159:401-10.

[12] Friedland W, et al. Track structures, DNA targets and radiation effects in the biophysical Monte Carlo simulation code PARTRAC. Mutat Res 2011;711:28-40.

[13] Friedland W, et al. Comprehensive track-structure based evaluation of DNA damage by light ions from radiotherapy-relevant energies down to stopping. Sci Rep 2017;7:45161.

[14] McNamara A, et al. Validation of the radiobiology toolkit TOPAS-nBio in simple
DNA geometries. Phys Med 2017;33:207-15.

[15] Nikjoo H, et al. Track-structure codes in radiation research. Radiat Meas 2006;41:1052-74.

[16] Meylan S, et al. Simulation of early DNA damage after the irradiation of a fibroblast cell nucleus using Geant4-DNA. Sci Rep 2017;7:11923.

[17] Rosales LF, et al. Accounting for radiation-induced indirect damage on DNA with the Geant4-DNA code. Phys Med 2018;51:108-16.

[18] Incerti S, et al. The Geant4-DNA project. Int J Model Simul Sci Comput 2010;1:157-78.

[19] Incerti S, et al. Comparison of Geant4 very low energy cross section models with experimental data in water. Med Phys 2010;37:4692-708.

[20] Bernal MA, et al. Track structure modeling in liquid water: a review of the Geant4 DNA very low energy extension of the Geant4 Monte Carlo simulation toolki. Phys Med 2015;31:157-78.

[21] Incerti S, et al. Geant4-DNA example applications for track structure simulations in liquid water: a report from the Geant4-DNA Project. Med Phys 2018;45:e722-39.

[22] Agostinelli S, et al. Geant4 — a simulation toolkit. Nucl Instrum Meth A 2003;506:250-303.

[23] Allison J, et al. Geant4 developments and applications. IEEE Trans Nucl Sci 2006;53:270-8.

[24] Allison J, et al. Recent developments in Geant4. Nucl Instrum Meth A 2016;835:186-225.

[25] Green NJB, et al. Stochastic modeling of fast kinetics in a radiation track. J Phys Chem 1990;94:251-8.

[26] Lampe N, et al. Mechanistic DNA damage simulations in Geant4-DNA part 1: a parameter study in a simplified geometry. Phys Med 2018;48:135-45.

[27] Lampe N, et al. Mechanistic DNA damage simulations in Geant4-DNA part 2: electron and proton damage in a bacterial cell. Phys Med 2018;48:146-55.

[28] Lampe N. The long term impact of ionising radiation on living systems [Ph.D thesis]. University Clermont-Auvergne; 2017.

[29] Ghosh SK, et al. How epigenome drives chromatin folding and dynamics, insights from efficient coarse-grained models of chromosomes. PLOS Comput Biol 2018;14:e1006159.

[30] Champion C, et al. Proton transport in water and DNA components: a Geant4 Monte Carlo simulation. Nucl Instrum Meth B 2013;306:165-8.

[31] Emfietzoglou D. Inelastic cross-sections for electron transport in liquid water: a comparison of dielectric models. Radiat Phys Chem 2003;66:373-85.

[32] Ivanchenko VN, et al. Recent improvements in Geant4 electromagnetic physics models and interfaces. Prog Nucl Sci Technol 2011;2:898-903.

[33] Kyriakou I, et al. The impact of new Geant4-DNA cross section models on electron track structure simulations in liquid water. J Appl Phys 2016;119:194902

[34] Kyriakou I, et al. Technical note: improvements in Geant4 energy-loss model and the effect on low-energy electron transport in liquid water. Med Phys 2015;42:3870-6.

[35] Bordage MC, et al. Implementation of new physics models for low energy electrons in liquid water in Geant4-DNA. Phys Med 2016;32(12):1833-40.

[36] Kyriakou I, et al. Microdosimetry of electrons in liquid water using the low-energy models of Geant4. J Appl Phys 2017;122:024303.

[37] Kreipl MS, et al. Time- and space-resolved Monte Carlo study of water radiolysis for photon, electron and ion irradiation. Radiat Environ Biophys 2009;48(1):11-20.

[38] Michalik V, et al. Computer-aided stochastic modeling of the radiolysis of liquid water. Radiat Res 1998;149(3):224-36.

[39] Davidkova M, et al. Predicting radiation damage distribution in biomolecules, in Radiation chemistry: from basics to applications in material and life sciences. Les Ulis, France: EDP Sciences; 2008.

[40] Karamitros M, et al. Introducing the Independent Reaction Time method in Geant4, Manuscript in preparation will be submitted to J. Comput. Phys.

[41] Karamitros M, et al. Modeling radiation chemistry in the Geant4 toolkit. Prog Nucl Sci Technol 2011;2:503-8.

[42] Karamitros M, et al. Diffusion-controlled reactions modeling in Geant4-DNA. J Comput Phys 2014;274:841-82.

[43] Frankenberg D, et al. Induction of DNA double-strand breaks by $1 \mathrm{H}$ and $4 \mathrm{He}$ ions in primary human skin fibroblasts in the LET range of 8 to $124 \mathrm{keV} / \mathrm{um}$. Radiat Res 1999;151:540-9.

[44] Campa A, et al. DNA DSB induced in human cells by charged particles and gamma rays: experimental results and theoretical approaches. Int J Radiat Biol 2005;81:841-54.

[45] Belli M, et al. DNA DSB induction and rejoining in V79 cells irradiated with light ions: a constant field gel electrophoresis study. Int J Radiat Biol 2000;76:1095-104.

[46] Leloup C, et al. Evaluation of lesion clustering in irradiated plasmid DNA. Int J Radiat Biol 2005;81:41-54.

[47] Paul TT, et al. A critical role for histone H2AX in recruitment of repair factors to nuclear foci after DNA damage. Curr Biol 2000;10:886-95.

[48] Kryndushkin DS, et al. Yeast [PSI + ] prion aggregates are formed by small Sup35 polymers fragmented by Hsp104. J Biol Chem 2003;278:49636-43.

[49] Iliakis G, et al. Measurement of DNA double strand breaks in $\mathrm{CHO}$ cells at various stages of the cell cycle using pulse field gel electrophoresis: calibrations by means of 125I decay. Int J Radiat Biol 1991;59:343-57.

[50] Berger MJ, et al. Stopping power and ranges for protons and alpha particles (report 49). J Int Commission Radiat Units Meas 1993;25(2):15.

[51] Belli M, et al. DNA Fragmentation in mammalian cells exposed to various light ions. Adv Space Res 2001;27:393-9. 